\title{
BOUNDED EARTHQUAKES
}

\author{
DRAGOMIR ŠARIĆ
}

(Communicated by Juha M. Heinonen)

\begin{abstract}
We give a short proof of the fact that bounded earthquakes of the unit disk induce quasisymmetric maps of the unit circle. By a similar method, we show that symmetric maps are induced by bounded earthquakes with asymptotically trivial measures.
\end{abstract}

\section{INTRODUCTION}

An earthquake $E$ of the unit disk $\mathbf{D}$ is a piecewise isometric (for the hyperbolic metric on $\mathbf{D})$ surjective self-map. The unit disk $\mathbf{D}$ is partitioned into nonintersecting geodesics and complementary ideal polygons, called the strata of $E$; $E$ is an isometry on each stratum. The set of geodesics in the strata is called the support of $E$ and it forms a geodesic lamination of $\mathbf{D}$. By the definition, an earthquake $E$ moves its strata to the left relative to each other. Thurston [12] introduced earthquakes of the unit disk and showed a remarkable fact that each homeomorphism of the unit circle $S^{1}$ can be obtained as an extension to the boundary $\partial \mathbf{D}=\mathbf{S}^{\mathbf{1}}$ of an earthquake map $E: \mathbf{D} \rightarrow \mathbf{D}[12$, Theorem 3.1].

Recall that a quasisymmetric map $h$ of the unit circle $S^{1}$ is an orientation preserving homeomorphism which distorts symmetric triples on $S^{1}$ in a bounded way, i.e. there exists $M>1$ such that $1 / M \leq|h(I)| /|h(J)| \leq M$ for all adjacent intervals $I, J \subset S^{1}$ with $|I| /|J|=1$ [1], [5]. A quasisymmetric map of $S^{1}$ extends to a quasiconformal map of the unit disk $\mathbf{D}$ [1]. Thus, an equivalent definition of a quasisymmetric map is that it maps quadruples on $S^{1}$ with positive, fixed cross-ratios (greater than 1) onto quadruples with cross-ratios uniformly bounded away from 1 and $\infty$. A symmetric map of $S^{1}$ is an orientation preserving homeomorphism of $S^{1}$ which maps symmetric triples of points on $S^{1}$ onto nearly symmetric triples of points as the size of the triples goes to zero, i.e. $|h(I)| /|h(J)| \rightarrow 1$ as $|I| \rightarrow 0$ for all $I, J \subset S^{1}$ adjacent intervals with $|I|=|J|[7]$, [11], [5]. For an equivalent definition in terms of quadruples of points on $S^{1}$ see [11] or proof of Theorem 2 in Section 4.

To an earthquake $E$ corresponds a unique transverse measure on its support (invariant under isotopies relative to the support geodesic lamination) given by measuring the relative movement to the left of the strata of $E$ [12]. Thurston [12] defined the norm of an earthquake measure to be the supremum over all geodesic

Received by the editors July 31, 2006.

2000 Mathematics Subject Classification. Primary 30F60, 30F45, 32H02, 32G05; Secondary $30 \mathrm{C} 62$.

Key words and phrases. Earthquakes, transverse bounded measures, asymptotically trivial measures.

(c) 2007 American Mathematical Society Reverts to public domain 28 years from publication 
$\operatorname{arcs} I$ of length 1 in $\mathbf{D}$ of the transverse measures deposited on $I$. If the norm is finite, then the earthquake measure is called bounded. The following theorem is suggested by Thurston's paper [12]:

Theorem 1. Let $\mu$ be an earthquake measure which defines an earthquake map $E_{\mu}$ whose extension to $S^{1}$ is a homeomorphism. Then the following are equivalent:

(1) $\mu$ is a bounded earthquake measure,

(2) $\left.E_{\mu}\right|_{S^{1}}: S^{1} \rightarrow S^{1}$ is a quasisymmetric map.

The first proof of the equivalence of the two conditions [10] involved a third condition:

(3) $\left.t \mapsto E_{t \mu}\right|_{S^{1}}(x)$, for $x \in S^{1}$ and $t>0$, extends to a holomorphic motion of $S^{1}$ on the Riemann sphere.

The direction $(2) \Longrightarrow(1)$ is elementary [10, Proposition 2.1]. The key idea for proving $(1) \Longrightarrow(2)$ is to show that $(1) \Longrightarrow(3)$ by extending a positive parameter $t$ to a complex parameter with a small imaginary part and showing that the resulting map is a holomorphic motion which gives $(3) \Longrightarrow(2)$. This involved a geometric argument and the use of a result by Mañé, Sad and Sullivan [9] on holomorphic motions. Epstein, Marden and Markovic [4] somewhat improved the lower bound on the size of the imaginary part of the complex parameter in their investigation of convex cores of plane domains.

At approximately the same time as in [10], Gardiner, $\mathrm{Hu}$ and Lakic [6] gave another proof of Theorem 1 using ordinary differential equations. Their proof is more elementary than in [10] (since it does not use holomorphic motions) but it is more computational and it does not show the analyticity of the path $\left.t \mapsto E_{t \mu}\right|_{S^{1}}$, as in [10], but only differentiability of $\left.t \mapsto E_{t \mu}\right|_{S^{1}}(x), x \in S^{1}$. Later on, another proof was given by $\mathrm{Hu}[8]$ which was more direct but which used more computations as well. Both [6], [8] consider stronger than quasisymmetric smoothness properties of circle homeomorphisms.

Since earthquakes are given by a geometric construction in very elementary terms on $\mathbf{D}$ (Thurston [12, Introduction]), one would hope to have a simple proof of $(1) \Longleftrightarrow(2)$ without much computation and using only elementary hyperbolic geometry. We give a surprisingly easy proof of the equivalence of the two conditions. The main difficulty is in proving $(1) \Longrightarrow(2)$. The idea is to assume on the contrary that the extension to $S^{1}$ of the earthquake map is not quasisymmetric. By the re-scalings of quadruples of points where the quasisymmetric constant tends to infinity, we obtain a sequence of maps which cannot converge to a homeomorphism. However, their corresponding earthquake measures are the re-scalings of the bounded earthquake measure $\mu$. Hence they are uniformly bounded and there exists a convergent subsequence whose limit is a bounded earthquake measure. This implies that the corresponding subsequence of earthquakes, when properly normalized, converges to a homeomorphism of $S^{1}$. The contradiction finishes the proof. The proof of part $(2) \Longrightarrow(1)$ from [10, Theorem 1] is briefly outlined for the benefit of the reader.

An appropriate extension of the above method allows us to characterize which earthquakes extend to symmetric maps of the unit circle. (We remark that there exists a constant $0<r_{0}<1$ such that the geodesics of a lamination intersecting a hyperbolic disk of radius $r_{0}$ are all transverse to a single diameter of the disk. Therefore, it is possible to assign a total transverse measure to such disks. See 
Section 2 for more details.) We say that a bounded earthquake measure $\mu$ is asymptotically trivial if $\sup _{D} \mu(D) \rightarrow 0$ as $\delta \rightarrow 0$, where the supremum is over all hyperbolic disks $D$ of a fixed radius $r_{0}$ with centers on the Euclidean distance at most $\delta$ to the boundary $S^{1}$ of the unit disk $\mathbf{D}$.

Theorem 2. Let $h: S^{1} \rightarrow S^{1}$ be a homeomorphism and let $E_{\mu}: \mathbf{D} \rightarrow \mathbf{D}$ be the earthquake map such that $\left.E_{\mu}\right|_{S^{1}}=h$. Then the following are equivalent:

(4) $h$ is a symmetric map,

(5) $\mu$ is an asymptotically trivial earthquake measure.

\section{Preliminaries}

Definition 2.1 ([12]). Let $\lambda$ be a geodesic lamination on D. A stratum of $\lambda$ is either a geodesic of $\lambda$ or a component of the complement of $\lambda$ in $\mathbf{D}$, if any. An earthquake $E$ with the support $\lambda$ is a surjective map $E: \mathbf{D} \rightarrow \mathbf{D}$ such that $E$ is a hyperbolic isometry when restricted to any stratum and, for any two strata $A$ and $B$, the comparison isometry

$$
\left.E\right|_{B} \circ\left(\left.E\right|_{A}\right)^{-1}
$$

is a hyperbolic translation whose axis weakly separates $A$ and $B$, and which translates $B$ to the left as seen from $A$.

An earthquake $E$ of $\mathbf{D}$ continuously extends to a homeomorphism of the boundary $\partial \mathbf{D}=\mathbf{S}^{\mathbf{1}}$. We denote by $\left.E\right|_{S^{1}}$ the extension. A remarkable fact is that any homeomorphism of $S^{1}$ is obtained as a boundary extension of an earthquake [12].

The translation length of $\left.E\right|_{B} \circ\left(\left.E\right|_{A}\right)^{-1}$ is the first approximation to the transverse measure deposited on a geodesic arc $I$ connecting $A$ to $B$ corresponding to the earthquake $E$. The transverse measure of $I$ is obtained by taking the limit of the sum of translation lengths of comparison isometries between finitely many consecutive strata intersecting $I$, as the maximum distance between consecutive strata goes to zero. The transverse measure of any geodesic arc is well-defined. Two homeomorphisms of $S^{1}$ have the same transverse measures (of all geodesic arcs) corresponding to their earthquakes if and only if one homeomorphism is equal to the post-composition with a hyperbolic isometry of the other homeomorphism (see $[12]$ ). (Note that transverse measures determine earthquakes and circle homeomorphisms uniquely up to a post-composition by a hyperbolic isometry of $D$. Thus, given a transverse measure, we need to normalize the earthquake by fixing the hyperbolic isometry.) The transverse measures of arcs are invariant under isotopies of D which preserve the support geodesic lamination $\lambda$. The family of the transverse measures to all geodesic arcs in $\mathbf{D}$ is called an earthquake measure.

Let $\mathcal{G}$ be the set of all unoriented geodesics of $\mathbf{D}$. Then $\mathcal{G}$ is homeomorphic to $\left(S^{1} \times S^{1}-\operatorname{diag}\right) / \sim$, where $(a, b) \sim(b, a)$. It is sometimes useful to think of transverse measures to the support geodesic laminations $\lambda$ as measures on the space of geodesics $\mathcal{G}$ whose support is a geodesic lamination $\lambda$. An earthquake measure $\mu$ is a positive Radon measure on $\mathcal{G}$ whose support is a geodesic lamination.

Let $I$ be an arbitrary geodesic arc of length 1 . Then $\mu(I)$ is the total measure of $I$ deposited by an earthquake measure $\mu$, or alternatively, $\mu(I)$ is the $\mu$-mass of all geodesics in $\mathbf{D}$ intersecting $I$. Thurston [12] introduced the norm of an earthquake measure $\mu$ by

$$
\|\mu\|:=\sup _{I} \mu(I)
$$


where the supremum is over all closed geodesic arcs $I$ of length 1 . An earthquake measure $\mu$ is said to be bounded if $\|\mu\|<\infty$.

Let $r_{0}>0$ be a fixed number smaller than the hyperbolic radius of the inscribed disk in an ideal hyperbolic triangle in $\mathbf{D}$. If $D$ is a hyperbolic disk with radius $r_{0}$, then the set of geodesics, from an arbitrary geodesic lamination of $\mathbf{D}$, which intersect $D$ also intersect a single hyperbolic arc of length $2 r_{0}$. We define the measure of $D$ deposited by an earthquake measure $\mu$ of $\mathbf{D}$ to be equal to the measure deposited on the arc which is transverse to the subset of support of $\mu$ which intersects $D$.

Let $D$ be an arbitrary hyperbolic disk in $\mathbf{D}$. Define $\delta(D)$ to be the Euclidean distance of the center of $D$ to the boundary $S^{1}$. A bounded earthquake measure $\mu$ is asymptotically trivial if

$$
\sup _{D, \delta(D) \leq t} \mu(D) \rightarrow 0
$$

as $t \rightarrow 0$, where the supremum is over all hyperbolic disks $D$ with radius $r_{0}$ whose distance to the boundary $\delta(D)$ is at most $t$.

An earthquake cocycle whose support is a geodesic lamination $\lambda$ is a map $E$ : $\mathbf{D} \times \mathbf{D} \rightarrow \mathbf{P S L}_{\mathbf{2}}(\mathbb{R})$ with the cocycle property $\left(E\left(z_{1}, z_{2}\right) \circ E\left(z_{2}, z_{3}\right)=E\left(z_{1}, z_{3}\right)\right.$ for all $\left.z_{1}, z_{2}, z_{3} \in \mathbf{D}\right)$ such that, given two strata $A$ and $B$ for $\lambda$, we have $E\left(z_{1}, z_{2}\right)=$ $E\left(w_{1}, w_{2}\right)$ whenever $z_{1}, w_{1} \in A$ and $z_{2}, w_{2} \in B$. In addition, $E\left(z_{1}, z_{2}\right)$ is required to be a hyperbolic translation with the axis weakly separating the strata $A$ and $B$ containing $z_{1}$ and $z_{2}$ and translating $B$ to the left as seen from $A$. Given an earthquake measure $\mu$ there exists a corresponding earthquake cocycle which defines a piecewise isometric, injective map $E: \mathbf{D} \rightarrow \mathbf{D}$ [3]. (If $E$ is surjective, then it is an earthquake map.) The earthquake obtained in this way has its measure equal to $\mu[3],[6],[10]$.

\section{THE WEAK CONVERGENCE}

Denote by $\mathcal{G}_{z}$, for $z \in \mathbf{D}$, the set of geodesics in $\mathbf{D}$ which contain $z$. Given $z, w \in \mathbf{D}$ denote by $[z, w]$ the geodesic arc in $\mathbf{D}$ between $z$ and $w$. If $K$ is a subset of $\mathbf{D}$, denote by $\mathcal{G}_{K}$ the set of geodesics of $\mathbf{D}$ which intersect $K$. The following lemma is essentially proved by Epstein and Marden [3, Theorem 3.11.5]. Their statement is for sequences of finite complex earthquake measures, but it immediately extends to arbitrary (not necessarily finite) sequences of positive earthquakes with a simpler proof.

Lemma 3.1 ([3]). Let $\mu_{i}, \mu$ be earthquake measures such that $\mu_{i} \rightarrow \mu$ as $i \rightarrow \infty$ in the weak* topology. Then the sequence $E_{\mu_{i}}\left(z_{1}, z_{2}\right)$, for any $z_{1}, z_{2} \in \mathbf{D}$, of earthquake cocycles has a convergent subsequence. Any limit $E\left(z_{1}, z_{2}\right)$ of a subsequence $E_{\mu_{i_{j}}}\left(z_{1}, z_{2}\right)$ satisfies

$$
E_{\mu}\left(z_{1}, z_{2}\right)=T_{g_{2}}^{a_{2}} \circ E\left(z_{1}, z_{2}\right) \circ T_{g_{1}}^{a_{1}},
$$

where $a_{k}=\mu\left(g_{k}\right)-\nu\left(g_{k}\right), \nu$ is the weak ${ }^{*}$ limit of $\mu_{i_{j}}$ restricted to $\mathcal{G}_{\left[z_{1}, z_{2}\right]}$, and $T_{g_{k}}^{a_{k}}$, $k=1,2$, is either the identity if $a_{k}=0$, or it is a hyperbolic translation with the axis $g_{k}\left(z_{k} \in g_{k}\right)$ in the support of $\mu$ and the translation length $a_{k}$.

In the following lemma we require that the sequence of earthquake measures $\mu_{i}$ is uniformly bounded. The proof appears to be somewhat subtle and it strongly uses the assumption of being uniformly bounded. (One could expect that the lemma should hold without the above assumption.) 
Lemma 3.2. Let $\mu_{i}, \mu$, for $i=1,2, \ldots$, be uniformly bounded earthquake measures (i.e. $\|\mu\|,\left\|\mu_{i}\right\| \leq M<\infty$ for all $i$ ) on the unit disk $\mathbf{D}$. If $\mu_{i} \rightarrow \mu$ as $i \rightarrow \infty$ in the weak* topology, then, for each $x \in S^{1}$,

$$
\left.\left.E_{\mu_{i}}\right|_{S^{1}}(x) \rightarrow E_{\mu}\right|_{S^{1}}(x)
$$

as $i \rightarrow \infty$, when the earthquakes $\left.E_{\mu_{i}}\right|_{S^{1}},\left.E_{\mu}\right|_{S^{1}}$ are properly normalized.

Proof. Recall that $\left.E_{\mu_{i}}\right|_{S^{1}},\left.E_{\mu}\right|_{S^{1}}$ are well-defined up to post-composition by a hyperbolic isometry of $\mathbf{D}$. We normalize the earthquake maps as follows. Either the earthquake measure $\mu$ has a complementary gap $A$ in $\mathbf{D}$ or the support of $\mu$ foliates D. In the first case, we fix $E_{\mu}$ to be the identity on the gap $A$. In the second case, we fix a geodesic $l$ in the support of $\mu$ such that $\mu(l)=0$ and set $\left.E_{\mu}\right|_{l}=i d$.

If the support of $\mu$ has a gap $A$, then either the support of $\mu_{i}$ has a gap $A_{i}$ which intersects $A$ with at least one boundary side contained in a fixed compact subset of $\mathcal{G}$, or there exists a geodesic $l_{i}$ in the support of $\mu_{i}$ of zero $\mu_{i}$-measure which intersects $A$ and is contained in the fixed compact subset of $\mathcal{G}$ (for $i$ large enough depending on the fixed compact subset of $\mathcal{G}$ ). If the support of $\mu$ has no gaps, then there exists a geodesic $l_{i}$ in the support of $\mu_{i}$ such that $\mu_{i}\left(l_{i}\right) \rightarrow 0$ and $l_{i} \rightarrow l$ as $i \rightarrow \infty$. Then we set either $\left.E_{\mu_{i}}\right|_{A_{i}}=i d$ or $\left.E_{\mu_{i}}\right|_{l_{i}}=i d$.

We fix $r, 0<r<1$, and define $\mu^{\prime}$ to be the restriction of $\mu$ to the set of geodesics which intersect the euclidean disk $\mathbf{D}_{\mathbf{r}}$ centered at 0 of radius $r$. We think of $\mu^{\prime}$ as a new earthquake measure on $G$ whose support geodesics intersect $\mathbf{D}_{\mathbf{r}}$. Then there exists a sequence $r_{i} \rightarrow r, r_{i} \geq r$, such that $\mu_{i}^{\prime} \rightarrow \mu^{\prime}$ as $i \rightarrow \infty$, where $\mu_{i}^{\prime}$ is the restriction of $\mu_{i}$ to the subset of $\mathcal{G}$ whose geodesics intersect $\mathbf{D}_{\mathbf{r}_{\mathbf{i}}}$. For $r$ large enough, either $A$ is contained in or is equal to a stratum $A^{\prime}$ of $\mu^{\prime}$, or $l$ is in the support of $\mu^{\prime}$. Then, for $i$ large enough, either $A_{i}$ is contained in a stratum $A_{i}^{\prime}$ of $\mu_{i}^{\prime}$, or $l_{i}$ is in the support of $\mu_{i}^{\prime}$. We normalize $E_{\mu^{\prime}}$ and $E_{\mu_{i}^{\prime}}$ in a corresponding manner.

We show that $\left.\left.E_{\mu_{i}^{\prime}}\right|_{S^{1}}(x) \rightarrow E_{\mu^{\prime}}\right|_{S^{1}}(x)$ as $i \rightarrow \infty$, for all $x \in S^{1}$. Since the supports of $\mu^{\prime}$ and $\mu_{i}^{\prime}$ are contained in a compact subset of $\mathcal{G}$, each $x \in S^{1}$ is on the boundary of at least one stratum of the measures $\mu^{\prime}$ and $\mu_{i}^{\prime}$. Note that $E_{\mu^{\prime}}$ has at most countably many leaves in the support with non-zero measure which intersect $S^{1}$ in at most countably many points. It is enough to prove the convergence outside these points of $S^{1}$ due to the fact that $\left.E_{\mu^{\prime}}\right|_{S^{1}}$ and $\left.E_{\mu_{i}^{\prime}}\right|_{S^{1}}$ are order preserving maps. If $x \in S^{1}$ is a point which lies on the boundary of a stratum $B$ of $\mu^{\prime}$ (if the stratum is a geodesic, then the $\mu$-measure is zero by our assumption), then the measures $\mu_{i}^{\prime}$ restricted to the geodesic arc $I$ connecting the fixed stratum $\left(A^{\prime}\right.$ or $\left.l^{\prime}\right)$ of $\mu^{\prime}$ to the stratum $B$ ( $I$ connects the interior of the stratum $A^{\prime}$ with the interior of the stratum $B$ if the strata are gaps) converge to the restriction of the measure $\mu^{\prime}$ to $I$. Thus the earthquake cocycles $E_{\mu_{i}^{\prime}}$ corresponding to the endpoints of $I$ converge to $E_{\mu^{\prime}}$ by Lemma 3.1. Moreover, since each geodesic in the support of $\mu^{\prime}$ is approximated by the geodesics of the support of $\mu_{i}^{\prime}$, there exists a sequence $I_{i}$ of closed geodesic arcs (which lie on the same ideal geodesic as $I$ ) with one endpoint at $A_{i}^{\prime}$ or $l_{i}^{\prime}$ and the other endpoint at $B_{i}$ (where $B_{i}$ is a stratum of $\mu_{i}^{\prime}$ converging to $B$ and containing $x \in S^{1}$ on its boundary) which converge toward $I$. It follows by our choice of $B_{i}$ that the restriction of $\mu_{i}^{\prime}$ to $I_{i}$ converges to the restriction of $\mu^{\prime}$ to $I$ (when both restrictions are considered as measures on $\mathcal{G}$ ) in the weak* topology. By Lemma 3.1, we get that the cocycles for $\mu_{i}^{\prime}$ with respect to the endpoints of $I_{i}$ converge to the cocycle for $\mu^{\prime}$ with respect to $I$ when they are considered as hyperbolic isometries of $\mathbf{D}$. This implies the desired convergence for any fixed $r<1$. 
Therefore, to show that $\left.\left.E_{\mu_{i}}\right|_{S^{1}}(x) \rightarrow E_{\mu}\right|_{S^{1}}(x)$ as $i \rightarrow \infty$, it is enough to show that $\left.\left.E_{\mu_{i}^{\prime}}\right|_{S^{1}}(x) \rightarrow E_{\mu_{i}}\right|_{S^{1}}(x)$ and $\left.\left.E_{\mu^{\prime}}\right|_{S^{1}}(x) \rightarrow E_{\mu}\right|_{S^{1}}(x)$ as $r \rightarrow 1$ independent of $i$. By the cocycle property, we have $\left.E_{\mu_{i}}\right|_{S^{1}}(x)=\left.\left.E_{\mu_{i}^{\prime}}\right|_{S^{1}} \circ E_{\mu_{i}^{\prime \prime}}\right|_{S^{1}}(x)$, where $E_{\mu_{i}^{\prime \prime}}$ is normalized to be the identity on the stratum $C$ which contains $\mathbf{D}_{\mathbf{r}}$ and $\mu_{i}^{\prime \prime}=\mu_{i}-\mu_{i}^{\prime}$. However $\left.E_{\mu_{i}}\right|_{S^{1}}(x)=\left.\left.E_{\tilde{\mu}_{i}^{\prime \prime}}\right|_{S^{1}} \circ E_{\mu_{i}^{\prime}}\right|_{S^{1}}(x)$, where $\tilde{\mu}_{i}^{\prime \prime}=\left(\left.E_{\mu_{i}^{\prime}}\right|_{S^{1}}\right)^{*}\left(\mu_{i}^{\prime \prime}\right)$ and $E_{\tilde{\mu_{i}}}{ }^{\prime \prime}$ is normalized to be the identity on the stratum $E_{\mu_{i}^{\prime}}(C)$. Note that the support of $\mu_{i}^{\prime \prime}$ does not intersect $\mathbf{D}_{\mathbf{r}}$ except possibly on the boundary $\partial \mathbf{D}_{\mathbf{r}}$, which implies that the diameter of each stratum of $\mu_{i}^{\prime \prime}$ outside $\mathbf{D}_{\mathbf{r}}$ goes to zero as $r \rightarrow 1$. The image of the support of $\mu_{i}^{\prime \prime}$ under $E_{\mu_{i}^{\prime}}$ is not increasing by much, namely its diameter also goes to zero as $r \rightarrow 1$. This follows by an easy observation that bounded earthquakes decrease the distance between their support geodesics by a bounded amount [12], [10, Lemma 2.1, Proposition 2.1]. Since the distance between $\left.E_{\mu_{i}^{\prime}}\right|_{S^{1}}(x)$ and $\left.E_{\mu_{i}}\right|_{S^{1}}(x)$ is at most the diameter of the support of $\tilde{\mu}_{i}^{\prime \prime}$, we get the desired convergence.

In the following proposition we show that the converse is true.

Proposition 3.3. Let $\mu, \mu_{i}$ be uniformly bounded earthquake measures on $\mathbf{D}$. Then $\mu_{i} \rightarrow \mu$ in the weak* topology as $i \rightarrow \infty$ if and only if there exist normalizations of earthquake maps $\left.E_{\mu_{i}}\right|_{S^{1}},\left.E_{\mu}\right|_{S^{1}}$ such that $\left.\left.E_{\mu_{i}}\right|_{S^{1}}(x) \rightarrow E_{\mu}\right|_{S^{1}}(x)$ for each $x \in S^{1}$, as $i \rightarrow \infty$.

Proof. The "if" part is proved in Lemma 3.2 above. We show the "only if" part.

We suppose that $\left.\left.E_{\mu_{i}}\right|_{S^{1}} \rightarrow E_{\mu}\right|_{S^{1}}$ pointwise as $i \rightarrow \infty$. Assume on the contrary that $\mu_{i}$ does not converge to $\mu$ in the weak* topology. By the compactness of the space of probability measures on a compact space, there exists a subsequence $\mu_{i_{j}}$ of $\mu_{i}$ which converges to a measure $\nu$ in the weak* topology (by the standard diagonal argument). It is clear that $\nu$ is an earthquake measure bounded by the same constant as the sequence $\mu_{i}$. Our assumption implies that $\nu \neq \mu$. By Lemma 3.2, there exist normalizations of $E_{\mu_{i_{j}}}$ and $E_{\nu}$ such that $\left.\left.E_{\mu_{i_{j}}}\right|_{S^{1}} \rightarrow E_{\nu}\right|_{S^{1}}$ pointwise, as $j \rightarrow \infty$. However, since $\left.\left.E_{\mu_{i}}\right|_{S^{1}} \rightarrow E_{\mu}\right|_{S^{1}}$ with possibly different normalization and since two normalizations differ by a post-composition with a hyperbolic isometry of $\mathbf{D}$, we conclude that $\left.E_{\mu}\right|_{S^{1}}=\left.\gamma \circ E_{\nu}\right|_{S^{1}}$ for some hyperbolic isometry $\gamma$. By the uniqueness of the earthquake measure [12], we have that $\nu=\mu$ which gives a contradiction. Therefore $\mu_{i} \rightarrow \mu$.

\section{Proofs of theOREMS}

Proof of Theorem 1. We sketch the proof of $(2) \Longrightarrow(1)$ from [10]. Assume on the contrary that $\mu$ is unbounded. If there is a sequence of geodesic $l_{i}$ in the support of $\mu$ whose measure $\mu\left(l_{i}\right) \rightarrow \infty$, then we find a sequence of quadruples with fixed crossratios whose images have unbounded cross-ratios. It is enough to take quadruples which have one point in each half-plane of $\mathbf{D}-\mathbf{l}_{\mathbf{i}}$ and the other two points to be the endpoints of $l_{i}$. Since the earthquake translates to the left and $\mu\left(l_{i}\right) \rightarrow \infty$, it follows that the cross-ratios of the images are unbounded. Thus the boundary map is not quasisymmetric. This is a contradiction. In the case that there is no such $l_{i}$ in the support of $\mu$, then we can find a sequence of subsets $\mathcal{L}_{i}$ of the support of $\mu$ whose geodesics each have one endpoint in an interval $\left(a_{i}, b_{i}\right)$ and the other endpoint in an interval $\left(c_{i}, d_{i}\right)$ with the cross-ratio of $\left(a_{i}, b_{i}, c_{i}, d_{i}\right)$ converging to infinity. Therefore, $\mathcal{L}_{i}$ is close to being a single geodesic and a similar argument applies. 
We prove $(1) \Longrightarrow(2)$. Assume on the contrary that $h:=\left.E_{\mu}\right|_{S^{1}}$ is not quasisymmetric. We already know that $h$ is a homeomorphism by [12], [10, Proposition 2.1], or [6]. Then there exists a sequence $\left(a_{i}, b_{i}, c_{i}, d_{i}\right)$ of counterclockwise oriented quadruplets of points on $S^{1}$ with cross-ratio 2 such that $\operatorname{cr}\left(h\left(a_{i}\right), h\left(b_{i}\right), h\left(c_{i}\right), h\left(d_{i}\right)\right)$ $\rightarrow \infty$ as $i \rightarrow \infty$. Let $A_{i}$ be the hyperbolic isometry such that $A_{i}:\left(a_{i}, b_{i}, c_{i}, d_{i}\right)$ $\mapsto(1, i,-1,-i)$. Then $h_{i}:=h \circ A_{i}^{-1}$ is a sequence of maps which are not quasisymmetric. Note that $\gamma_{i} \circ h_{i}=\left.E_{\mu_{i}}\right|_{S^{1}}$ for some hyperbolic isometry $\gamma_{i}$, where $\mu_{i}:=\left(A_{i}\right)^{*}(\mu)$. Since $\mu_{i}$ is a sequence of uniformly bounded earthquake measures, there is a subsequence $\mu_{i_{j}}$ which converges to a bounded earthquake measure $\sigma$. By Proposition 3.3, we get that $\left.\left.E_{\mu_{i j}}\right|_{S^{1}} \rightarrow E_{\sigma}\right|_{S^{1}}$ pointwise as $j \rightarrow \infty$ when the earthquakes are properly normalized.

On the one hand, $\left.E_{\mu_{i_{j}}}\right|_{S^{1}}=\gamma_{i_{j}} \circ h_{i_{j}}$, where $\gamma_{i_{j}}$ is a hyperbolic isometry of D. On the other hand, $\left.E_{\mu_{i_{j}}}\right|_{S^{1}}$ converges to a homeomorphism of $S^{1}$ which implies that the cross-ratios of $\left.E_{\mu_{i_{j}}}\right|_{S^{1}}(1, i,-1,-i)$ are uniformly bounded. But this is in contradiction with $\operatorname{cr}\left(h\left(a_{i}\right), h\left(b_{i}\right), h\left(c_{i}\right), h\left(d_{i}\right)\right) \rightarrow \infty$ as $i \rightarrow \infty$ because $\operatorname{cr}\left(h\left(a_{i_{j}}\right), h\left(b_{i_{j}}\right), h\left(c_{i_{j}}\right), h\left(d_{i_{j}}\right)\right)=c r\left(h_{i_{j}}(1), h_{i_{j}}(i), h_{i_{j}}(-1), h_{i_{j}}(-i)\right)$. Thus $h$ is quasisymmetric.

Proof of Theorem 2. We first show the $(4) \Longrightarrow(5)$ part. Assume on the contrary that $\mu$ is not an asymptotically trivial earthquake measure. Then there exists a sequence of hyperbolic disks $D_{i}$ whose radii are $r_{0}$ and whose centers are $d_{i} \in \mathbf{D}$, $\left|d_{i}\right| \rightarrow 1$, such that $\mu\left(D_{i}\right) \geq m>0$. Let $A_{i}$ be the hyperbolic translation whose axis is the geodesic of $\mathbf{D}$ through $d_{i}$ and 0 such that $A_{i}: d_{i} \mapsto 0$. Let

$$
\mu_{i}:=A_{i}^{*}(\mu)
$$

and let $D$ be the hyperbolic disk with center 0 and radius $r_{0}$.

Since $\left\|\mu_{i}\right\|=\|\mu\|$, for each $i$, it follows that the sequence of positive earthquake measures $\mu_{i}$ is uniformly bounded. Then there exists a convergent subsequence $\mu_{i_{j}} \rightarrow \sigma$ as $j \rightarrow \infty$ in the weak* topology, where $\sigma$ is a bounded earthquake measure. Note that $\sigma(D) \geq m>0$ because $\mu_{i_{j}}(D)=\mu\left(D_{i_{j}}\right) \geq m$. By Proposition 3.3 , there exists a normalization of earthquakes $E_{\sigma}, E_{\mu_{i_{j}}}$ such that $\left.\left.E_{\mu_{i_{j}}}\right|_{S^{1}} \rightarrow E_{\sigma}\right|_{S^{1}}$ pointwise as $j \rightarrow \infty$. In fact, it follows from the proof that we can normalize $E_{\sigma}$ to be the identity on a stratum intersecting $D$. Since $\sigma \neq 0$, we have that $h^{*}:=\left.E_{\sigma}\right|_{S^{1}}$ is not the restriction to $S^{1}$ of a hyperbolic isometry of $\mathbf{D}$ by the uniqueness of the earthquake measures [12]. Define $h_{i_{j}}:=\left.E_{\mu_{i_{j}}}\right|_{S^{1}}$.

Let $f^{*}:=\operatorname{ex}\left(h^{*}\right), f_{i_{j}}:=e x\left(h_{i_{j}}\right)$ and $f:=e x(h)$ be barycentric extensions (see [2]) of $h^{*}, h_{i_{j}}$ and $h$, respectively. Since $h$ is symmetric, it follows that $f$ is asymptotically conformal, i.e. given any $\epsilon>0$ there exists a compact subset $K$ of $\mathbf{D}$ such that the supremum of the absolute value of the Beltrami coefficient of $f$ over $\mathbf{D}-\mathbf{K}$ is at most $\epsilon$. Note that $f_{i_{j}} \rightarrow f^{*}$ pointwise by the properties of barycentric extension [2] because $h_{i_{j}} \rightarrow h^{*}$. Moreover, since $h_{i_{j}}$ is obtained as an extension to the boundary of the earthquake $E_{\mu_{i_{j}}}$ whose measure is the push-forward of the measure for $h$ by the hyperbolic isometry $A_{i_{j}}$, we get that

$$
h_{i_{j}}=B_{i_{j}} \circ h \circ A_{i_{j}}^{-1},
$$

where $B_{i_{j}}$ is a hyperbolic isometry. Since barycentric extension is conformaly natural, we get

$$
f_{i_{j}}=B_{i_{j}} \circ f \circ A_{i_{j}}^{-1} .
$$


By taking Beltrami coefficients of the left and of the right side in the above equation, we get that

$$
\operatorname{Belt}\left(f_{i_{j}}\right)=\operatorname{Belt}(f) \circ A_{i_{j}}^{-1} \frac{\overline{A_{i_{j}}^{-1}}}{A_{i_{j}}^{-1}} .
$$

Since $f$ is asymptotically conformal and by the definition of $A_{i_{j}}$, we conclude that $\operatorname{Belt}(f) \circ A_{i_{j}}^{-1} \rightarrow 0$ as $j \rightarrow \infty$ uniformly on a compact subset of $\mathbf{D}$. Thus $f^{*}$ is a hyperbolic isometry of $\mathbf{D}$ and its extension $h^{*}$ to the boundary is necessarily a hyperbolic isometry. Contradiction. Therefore $\mu$ is asymptotically trivial.

We show the $(5) \Longrightarrow(4)$ part. Assume on the contrary that $h=\left.E_{\mu}\right|_{S^{1}}$ is not symmetric. We recall a characterization of symmetric maps from [11]. Cover $S^{1}$ by finitely many charts which are closed, bounded intervals in $\mathbb{R}$. In each chart, a 4-tuple $(a, b, c, d), a, b, c, d \in \mathbb{R}$, is called standard if $|a-b|=|b-c|=|c-d|$. A homeomorphism of $S^{1}$ is symmetric if and only if, for any standard 4-tuple $(a, b, c, d)$ in any chart, the cross-ratio distortion $\log \frac{\operatorname{cr}(h(a, b, c, d))}{c r(a, b, c, d)}$ of $h$ converges to 0 as $|a-b| \rightarrow 0$. (Any standard 4-tuple on $\mathbb{R}$ has cross-ratio $4 / 3$. We choose the chart maps for $S^{1}$ to be Möbius. Then the standard 4-tuples in charts are the images of 4 -tuples on $S^{1}$ with fixed cross-ratios $4 / 3$. By abuse of notation, we identify standard 4-tuples in charts with their images on $S^{1}$ under chart maps.)

Since $h$ is not symmetric, there exists a sequence of standard 4 -tuples $\left(a_{i}, b_{i}, c_{i}\right.$, $\left.d_{i}\right)$, with $\left|a_{i}-b_{i}\right| \rightarrow 0$, in a fixed chart such that $\log \frac{\operatorname{cr}\left(h\left(a_{i}, b_{i}, c_{i}, d_{i}\right)\right)}{\operatorname{cr}\left(a_{i}, b_{i}, c_{i}, d_{i}\right)}$ does not converge to 0 . Let $A_{i}$ be the hyperbolic isometry of $\mathbf{D}$ which maps $\left(a_{i}, b_{i}, c_{i}, d_{i}\right)$ onto $(-1, a, b, 1)$ with $\operatorname{cr}(-1, a, b, 1)=4 / 3$. Define $\mu_{i}:=A_{i}^{*}(\mu)$. Note that $\mu_{i} \rightarrow$ 0 as $i \rightarrow \infty$ in the weak ${ }^{*}$ topology because $\mu$ is asymptotically trivial. Then $\left.E_{\mu_{i}}\right|_{S^{1}} \rightarrow i d$ pointwise by Proposition 3.3 , when $\left.E_{\mu_{i}}\right|_{S^{1}}$ are properly normalized. Define $h_{i}:=h \circ A_{i}^{-1}$. Then $\left.E_{\mu_{i}}\right|_{S^{1}}=B_{i} \circ h_{i}$, for some hyperbolic isometry $B_{i}$. Since $B_{i} \circ h_{i} \rightarrow i d$ pointwise, we conclude that $\log \frac{\operatorname{cr}\left(B_{i} \circ h_{i}(-1, a, b, 1)\right)}{c r(-1, a, b, 1)} \rightarrow 0$ as $i \rightarrow \infty$. On the other hand, $\log \frac{c r\left(B_{i} \circ h_{i}(-1, a, b, 1)\right)}{c r(-1, a, b, 1)}=\log \frac{\operatorname{cr}\left(h_{i}(-1, a, b, 1)\right)}{\operatorname{cr}(-1, a, b, 1)}=\log \frac{\operatorname{cr}\left(h\left(A_{i}^{-1}(-1, a, b, 1)\right)\right)}{\operatorname{cr}(-1, a, b, 1)}=$ $\log \frac{c r\left(h\left(a_{i}, b_{i}, c_{i}, d_{i}\right)\right)}{\operatorname{cr}(-1, a, b, 1)}$ does not converge to 0 by the above. Contradiction. Therefore $h$ is symmetric.

\section{REFERENCES}

[1] L. V. Ahlfors, Lectures on Quasiconformal Mappings, D. Van Nostrand Company, Inc., Princeton, New Jersey, 1966. MR0200442 (34:336)

[2] A. Douady and C. J. Earle, Conformally natural extension of homeomorphisms of the circle, Acta Math. 157 (1986), no. 1-2, 23-48. MR857678 (87j:30041)

[3] D. B. A. Epstein and A. Marden, Convex hulls in hyperbolic space, a theorem of Sullivan and measured pleated surfaces, LMS Lecture Notes 111, pages 112-253. Cambridge University Press, 1987. MR903852 (89c:52014)

[4] D. B. A. Epstein, A. Marden and V. Markovic, Quasiconformal homeomorphisms and the convex hull boundary, Ann. of Math. 159 (2004), no. 1, 305-336. MR2052356 (2005d:30067)

[5] F. Gardiner and N. Lakic, Quasiconformal Teichmüller Theory, Mathematical Surveys and Monographs, Volume 76, A.M.S. 2000.

[6] F. Gardiner, J. Hu, and N. Lakic, Earthquake curves. In complex manifolds and hyperbolic geometry (Guanajuato, 2001), 141-195; and Contemp. Math., 311, Amer. Math. Soc., Providence, RI, 2002. MR1940169 (2003i:37033)

[7] F. Gardiner and D. Sullivan, Symmetric and quasisymmetric structures on a closed curve, Amer. J. of Math. 114 (1992), no. 4, 683-736. MR1175689 (95h:30020) 
[8] J. Hu, Earthquake measure and cross-ratio distortion, In the tradition of Ahlfors and Bers, III, 285-308, Contemp. Math., 355, Amer. Math. Soc., Providence, RI, 2004. MR2145070 (2006m:37056)

[9] R. Mañé, P. Sad and D. Sullivan, On the dynamics of rational maps, Ann. Sci. Ecole Norm. Sup. 16 (1983), 193-217. MR732343 (85j:58089)

[10] D. Šarić, Real and complex earthquakes, Trans. Amer. Math. Soc. 358 (2006), no. 1, 233-249. MR2171231 (2006i:30063)

[11] D. Sullivan, Bounds, quadratic differentials, and renormalization conjectures, Mathematics into the Twenty-first Century, Volume 2, Amer. Math. Soc., Providence, RI, 1992. MR1184622 (93k:58194)

[12] W. Thurston, Earthquakes in two-dimensional hyperbolic geometry. In Low-dimensional Topology and Kleinian Groups, ed. by D. B. A. Epstein, Warwick and Durham, 1984; L. M. S. Lecture Note Series 112, Cambridge University Press, Cambridge, 1986, 91-112. MR903860 (88m:57015)

[13] "The geometry and topology of 3-manifolds", Princeton University Lecture Notes, online at http://www.msri.org/publications/books/gt3m.

Institute for Mathematical Sciences, Stony Brook University, Stony Brook, New YORK 11794-3660

E-mail address: saric@math.sunysb.edu

Current address: Department of Mathematics, Queens College of CUNY, 65-30 Kissena Blvd., Flushing, New York 11367

E-mail address: dragomir.saric@qc.cuny.edu 